\title{
Behavioral Modeling Approach for Array of Amplifiers in Active Antenna Array System
}

\author{
Saeed Farsi*, John Dooley**, Keith Finnerty**, Dominique Schreurs*, Bart Nauwelaers* and \\ Ronan Farrell** \\ *Katholieke Universiteit Leuven, Leuven, Belgium \\ ** National University of Ireland Maynooth, Co. Kildare, Ireland
}

\begin{abstract}
Memory polynomial behavioral models are widely used to model nonlinear distortion mechanisms in a single RF power amplifier. However, when a number of power amplifiers are used in parallel, as in the case of active antenna arrays, the total number of parameters will increase significantly. Hence, new strategies are required to model and linearize these systems efficiently. In this paper a novel efficient modeling approach for these array systems is presented and experimentally verified by applying the method to the measured data of an array of amplifiers. The experimental results prove the efficiency of the approach.
\end{abstract}

Index Terms - Power amplifiers, behavioural model, memory polynomial, nonlinear circuits, active antenna arrays.

\section{INTRODUCTION}

The adoption of active antenna arrays (AAA) or smart antennas have the potential to significantly enhance the capacity and efficient use of spectrum and power in wireless communication systems compared to the existing methods. Although the underlying principles behind the technology are well established and have been used in radar and sonar applications for many years, it is only now that the processing power and antenna technology has reached a point where it is becoming commercially viable. With this change in application area there is a corresponding shift from high to low cost production. This shift has implications for component tolerances and manufacturing quality for the basestation. As a result there are expected to be variations in the characteristic performance of each transceiver chain. Furthermore, AAA basestations are designed with the intention of having all electronic components housed in a single enclosure and mounted at a height directly behind the radiating elements. If one power amplifier (PA) of, for instance, a 4-by-4 array were to fail and needed to be replaced, it is not feasible to have a replacement that can exactly match the characteristics of the other 15 [1]. Moreover, there will be a temperature difference even within the AAA enclosure since the preference is that these systems should use passive cooling techniques. Thus, heat energy will not have an even distribution inside the enclosure which leads to uneven temperature distribution throughout the AAA, resulting in some variations in amplifier responses. Hence, there are many sources of variations in the amplifiers of an AAA. Therefore, a solution is needed to provide first the ability to model and then the capability to compensate for variations in the multiple signal paths such that the resulting beam pattern is transmitted as intended and the AAA is not adversely affected by the variation in power amplifiers or by replacing power amplifiers.

Taken individually, each power amplifier can be characterized to a suitable accuracy by a memory polynomial model $[2,3]$. This class of models has proved to have high capabilities in accurate modeling of nonlinear circuits with nonlinear memory effects $[3,4]$.

This paper proposes a novel approach to efficiently model an array of amplifiers. It is composed of a core model along with differential models as many as the amplifiers. Due to the simplifications made to the model, it uses a very compact set of parameters which highly simplifies the training phase of modeling the complete array. This approach can also be used in linearization to reduce the total number of parameters, resulting in a much simpler training phase for the whole array.

The remainder of the paper is as follows. In Section II we explain the modeling approach adopted when considering the issues related to a typical AAA structure. Following, Section III presents the case of four experimentally measured power amplifiers which have different characteristics. Two model approaches are extracted for this test case and compared. Finally the conclusions drawn from these tests are presented.

\section{MODELLING APPROACH}

In this paper we investigate two modeling approaches for active antenna arrays. One approach is to use identical memory polynomial models [2] for all PAs. The second is to provide one core memory polynomial model [2] in common for all the PAs, along with differential blocks for each PA. In this way the relative accuracy of the two approaches and the relative size for the models can be determined for a typical scenario.

In an m-by-n antenna array of identical active elements, there are $m \times n$ amplifiers of which may have variations in their characteristics, as discussed in previous section. If identical models are used for all the amplifiers, the predicted outputs cannot be modeled well due to the differences discussed previously. Hence, there is an inevitable need for modeling each amplifier separately. On the other hand, if totally separate models are used for this purpose, the number of model parameters becomes large and it can make the approach 
inefficient. Thanks to the similarity of the array amplifiers, some simplifications can be applied which lead to considerably lower number of parameters. The approaches are investigated using a 2-by-2 amplifier array as an example. The same strategy can be applied for a higher number of array elements. The models used in this paper are all in the complex envelope domain. Note that crosstalk effects among the amplifiers are beyond the scope of this paper.

\section{A. Using identical models for all amplifiers}

Fig. 1 shows identical models used for modeling the four amplifiers. In this method the inputs of the amplifiers encounter the same model. This means that if the inputs are identical, the predicted outputs of the amplifiers will be the same, though it is not true in practice due to inherent differences between the amplifiers. These differences lead to considerable errors in array modeling, as will be shown later in the experimental results.

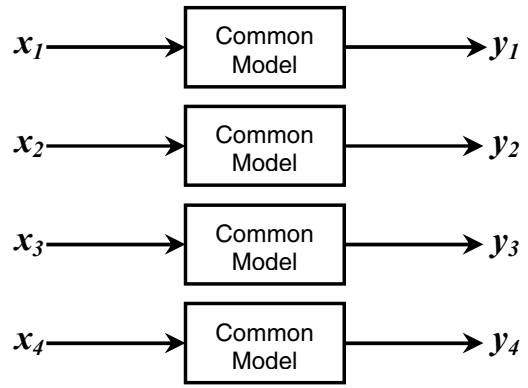

Fig. 1. Array of four amplifiers modeled with a common model

\section{B. Using Common-Differential Array Model (CDAM)}

Fig. 2 shows the proposed common-differential array model (CDAM). It is composed of a core model (CM) which is common in all the amplifiers, and four differential models (DMs) corresponding to four PAs which model the differences between the amplifiers. Since the DMs model the differential behavior of the array, namely that part of the behavior which is not modeled by the CM, their complexity is less than that of the common model. Therefore the overall array model constructed from using this approach is simpler than an array model made out of four identical models.

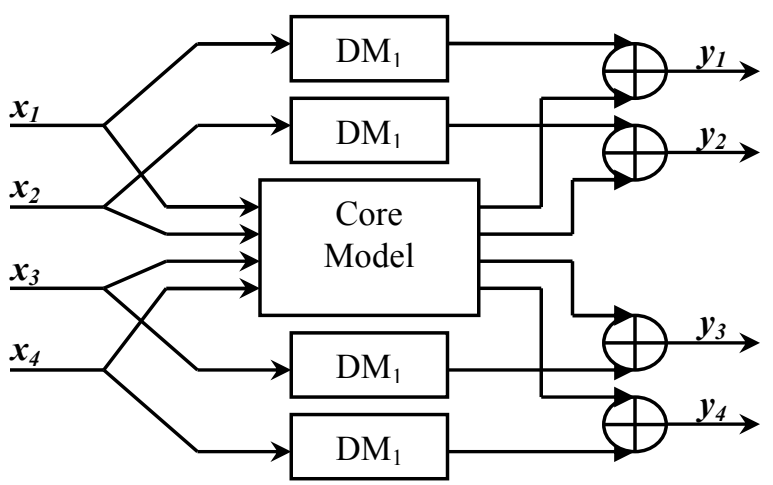

Fig. 2. Array of four amplifiers modeled with CDAM

In the next section, the experimental results of the two modeling approaches are demonstrated and discussed.

\section{EXPERIMENTAL RESULTS}

The four amplifiers used in this study are class $A B$ power amplifiers using ATF52189 from Avago Technologies [5]. These power amplifiers have been used in a testbed where the calibration of the separate AAA paths has been demonstrated [6]. For this reason the primary focus of the paper is not the calibration of the antennas but the modeling of the differences between power amplifier responses. To this end a testbench using Rohde \& Schwarz SMU 200A and FSQ is used to generate the PA input signal and to capture the output signals respectively. An identical input signal dataset of a single channel WCDMA waveform at $2.4 \mathrm{GHz}$ is used to measure each power amplifier output response. The instantaneous output power reaches the $2 \mathrm{~dB}$ compression point of the PAs. The four complex envelope responses are used to extract the coefficients for both modeling approaches.
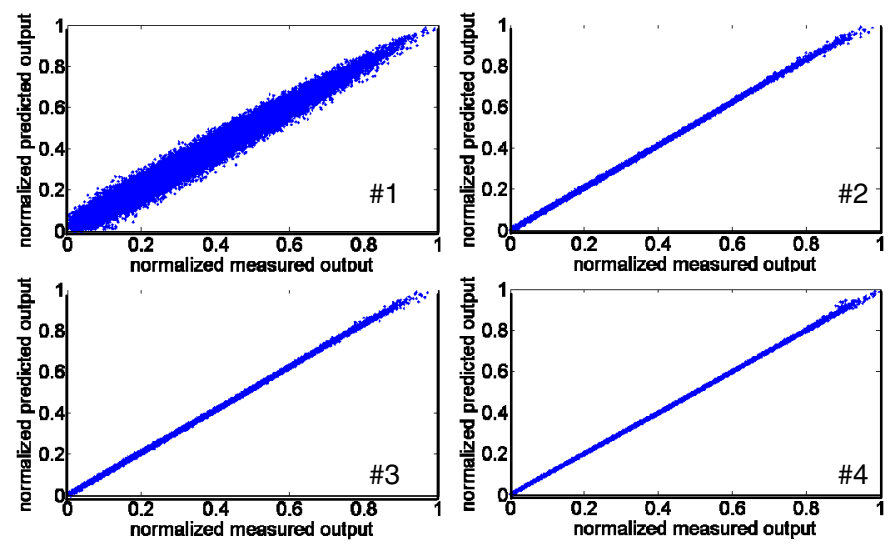

Fig. 3. Regression curves of an array of four amplifiers modeled with a common model

Fig. 3 illustrates the limited predicting ability of the first modeling approach to predict the outputs. In this figure, the predicted outputs by the models are plotted against the measured outputs. In these sets of measured data, the same sample sequence of modulated signal with the same power level has been used for all amplifiers. However since they are measured at different times, the inputs are not necessarily synchronized and identical. The memory polynomial model of the fourth amplifier has been used as the common model. A quantitative evaluation of the model is shown in Table 1. It illustrates the normalized mean square error (NMSE) values for the two approaches. As can be seen in Fig. 3, Fig. 4 and also in Table I, in the first approach the modeled output does not properly match the measured output for the other three PAs. In this particular case, a big difference between the amplifiers is the latency of the amplifiers resulting in large scattering of the data in Fig. 3. Nevertheless, it is not only this latency which leads to the differences, but also other factors such as those addressed in Section I, have considerable effects. Fig. 4 is a proof of this fact, since if it was only the effect of latency, no differences would be seen in the spectrum plot. For instance, the plots for amplifier \#1 in Fig. 4 show some mismatches between the target output and the predicted output calculated from the common model. This mismatch is not a 
simple shift caused by a simple gain difference and is frequency-dependent. This difference cannot be explained by a simple latency and/or gain difference.

A certain amount of spread can be seen at the upper end of the plots in Figs. 3 and 5. It is mainly because the output power reaches the high $2 \mathrm{~dB}$ compression point and modeling is difficult in this region.

In Fig. 2, as regarding the second modeling approach, the utilized core model is a memory polynomial with nonlinearity order of 6 and memory depth of 3 . The differential model is a linear model with memory depth of 2 . Using this linear differential model with low memory depth, remarkable improvement can be achieved. Note that the differential mode is not necessarily linear, and can be a higher-order nonlinear block, depending on the amount of nonlinearity and the amount of variations among the amplifiers. However in this special case a linear differential model is sufficient for desired accuracy.
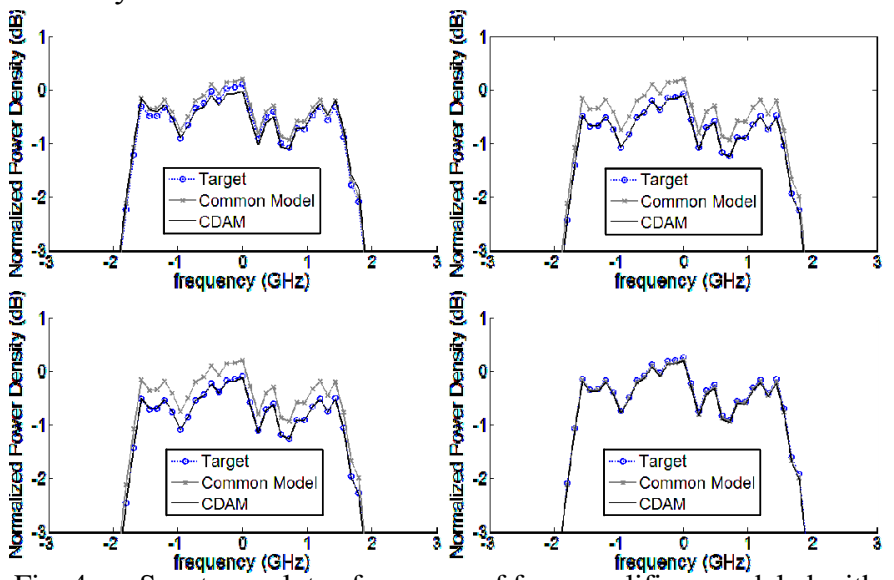

Fig. 4. Spectrum plots of an array of four amplifiers modeled with CDAM
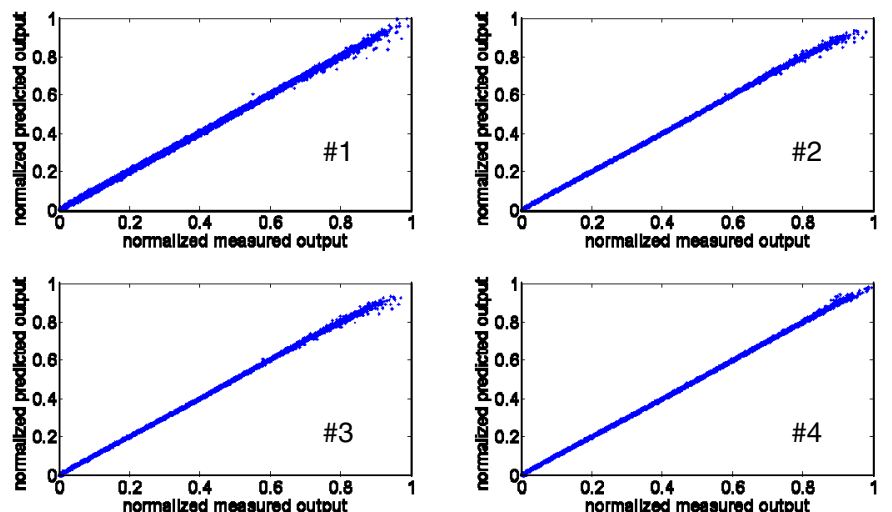

Fig.5. Regression curves of an array of four amplifiers modeled with CDAM

The results are shown in Fig. 4 and Fig. 5. They show highly acceptable performance of the proposed CDAM. The regression plots of Fig. 5 have less scattered points and are more linear as compared to those in Fig. 3. Note that since the error of using the common model is negligible in out-of-band response, it is not shown in Fig.4, and the figure is zoomed along the $y$-axis on the main band. The main variances in the amplifier responses are seen in the in-band spectrum, as in Fig. 4. In this figure it can be seen that the CDAM highly outperforms the common model, and is able to compensate the variations very well. A quantitative evaluation is shown in Table 1. This table proves the accuracy of the proposed method.

It is worth mentioning that the total number of parameters for the model in Fig. 2 is 34, while if four separate models were to be used the total number would be 72 . The difference will be more pronounced if a 4-by-4 array is considered.

TABLE I

NMSE VALUES FOR DIFFERENT AMPLIFIERS WITH CDAM

\begin{tabular}{|c|c|c|}
\hline \multirow{2}{*}{ Amplifier No. } & \multicolumn{2}{|c|}{ NMSE (dB) } \\
\cline { 2 - 3 } & Identical Models & CDAM \\
\hline 1 & -14.0 & -32.9 \\
\hline 2 & -22.1 & -37.2 \\
\hline 3 & -22.5 & -31.7 \\
\hline 4 & -36.1 & -36.1 \\
\hline
\end{tabular}

\section{CONCLUSIONS}

It is shown that the use of an identical model is not accurate for AAA systems where there exist variations between the PAs. In this paper a common-differential array model is proposed which can accurately and efficiently model variations between PAs in AAA systems with a highly reduced number of parameters. It consists of a common core block, modeling the common behavior of the PAs, plus several differential blocks which model the variances of the PAs. The model has been experimentally verified and proved its reliable performance.

\section{ACKNOWLEDGMENTS}

The authors acknowledge the financial support by FWOFlanders, KU Leuven GOA projects, and the Centre for Telecommunications Research (CTVR) and Science Foundation Ireland Grant 10/CE/I1853.

\section{REFERENCES}

[1] T. Cooper and R. Farrell, "Value-Chain Engineering of a TowerTop Cellular Base Station System", IEEE Vehicular Technology Conference, pp. 3184-3188, April 2007.

[2] J. Kim and K. Konstantinou, "Digital predistortion of wideband signals based on power amplifier model with memory," Electronics Letters, vol. 37, no. 23, pp. 1417-1418, November 2001.

[3] D. R. Morgan, Z. Ma, J. Kim, M. G. Zierdt and J. Pastalan, “A generalized memory polynomial model for digital predistortion of RF power amplifiers," IEEE Trans Signal Processing, vol. 54, no. 10 , pp. 3852-3860, October 2006.

[4] A. S. Tehrani, H. Cao, S. Afradoust, T. Eriksson, M. Isaksson, and C. Fager, "A comparative analysis of the complexityaccuracy tradeoff in power amplifier behavioral models," IEEE Trans. Microwave Theory \& Tech., vol. 58, no. 6, pp. 15101520, June 2010.

[5] L. Lengier, "An Antenna Array Testbed for Tower-Top Calibration", Masters Thesis, 2007.

[6] J. McCormack, T. Cooper and R. Farrell, "Tower-Top Antenna Array Calibration Scheme for Next Generation Networks", Eurasip Journal on Wireless Communications and Networking, vol. 2007, no. 2, pp. 1-12, January 2007. 